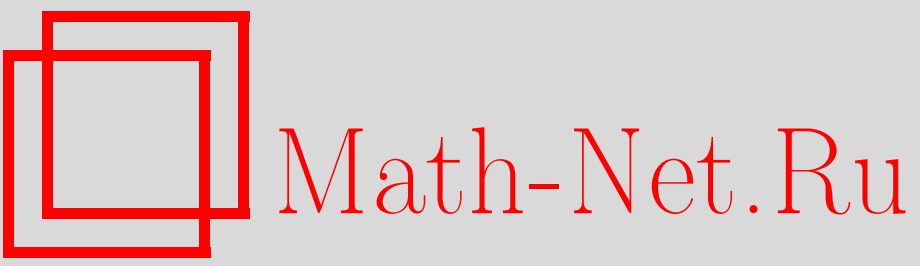

И. А. Высоцкая, Почти периодические на бесконечности решения разностных уравнений, Итоги науки и техн. Сер. Соврем. мат. и ее прил. Темат. обз., 2019, том 171, 38-46

DOI: https://doi.org/10.36535/0233-6723-2019-171-38-46

Использование Общероссийского математического портала Math-Net.Ru подразумевает, что вы прочитали и согласны с пользовательским соглашением

http://www. mathnet.ru/rus/agreement

Параметры загрузки:

IP : 54.162 .27 .143

26 апреля 2023 г., 18:34:34 


\title{
ПОЧТИ ПЕРИОДИЧЕСКИЕ НА БЕСКОНЕЧНОСТИ РЕШЕНИЯ РАЗНОСТНЫХ УРАВНЕНИЙ
}

\author{
(c) 2019 г. $\quad$ И. А. ВЫСОЦКАЯ
}

\begin{abstract}
АннотАция. Рассмотрен новый класс почти периодических на бесконечности функций, определяемый с помощью подпространства интегрально убывающих на бесконечности функций. Сформулированы четыре определения почти периодических на бесконечности функций и доказана их эквивалентность. Получены спектральные критерии почти периодичности на бесконечности ограниченных решений систем линейных разностных уравнений и их асимптотическое представление.
\end{abstract}

Ключевые слова: почти периодическая на бесконечности функция, медленно меняющаяся на бесконечности функции, интегрально убывающая на бесконечности функция, разностное уравнение.

\section{ALMOST PERIODIC AT INFINITY SOLUTIONS OF DIFFERENCE EQUATIONS}

\section{(c) 2019 I. A. VYSOTSKAYA}

\begin{abstract}
We consider a new class of functions almost periodic at infinity defined by using the subspace of functions that integrally decrease at infinity. We propose four definitions of functions almost periodic at infinity and prove their equivalence. Also, we obtain spectral criteria of almost periodicity at infinity of bounded solutions of systems of linear difference equations and their asymptotic representation.
\end{abstract}

Keywords and phrases: function almost periodic at infinity, function slowly changing at infinity, function integrally decreasing at infinity, difference equation.

AMS Subject Classification: 39A24

1. Введение. Проблема почти периодичности ограниченных функций на вещественный оси $\mathbb{R}=(-\infty,+\infty)$ и полуоси $\mathbb{R}_{+}=[0, \infty)$ как решений дифференциальных, разностных и функциональных уравнений изучалась многими авторами (см. $[5,9,15,16])$. Одной из первых работ в этом направлении исследований была статья С. Бохнера и Дж. фон Неймана [17], в которой установлена почти периодичность ограниченных решений разностных уравнений. С. Л. Соболевым был получен критерий почти периодичности решений параболических уравнений с самосопряженным оператором в гильбертовом пространстве (см. [12]).

В этой статье вводится в рассмотрение семейство исчезающих (в том или ином смысле) на бесконечности подпространств из банахова пространства непрерывных ограниченных на промежутке $\mathbb{J} \in\left\{\mathbb{R}_{+}, \mathbb{R}\right\}$ функций. По соответствующему выбранному подпространству исчезающих на бесконечности функций даются четыре эквивалентных (см. теорему 2.1) определения почти периодических на бесконечности функций.

При исследовании почти периодических на бесконечности функций существенно используется спектральная теория изометрических представлений, излагаемая в $[2,5]$. 
Основной результат статьи содержится в леммах 3.1, 3.2 и теореме 3.1. В этих утверждениях получены спектральные критерии почти периодичности на бесконечности ограниченных решений систем линейных разностных уравнений и их асимптотическое представление.

2. Основные понятия из теории почти периодических функций. Введем в рассмотрение основные функциональные пространства и сформулируем основные понятия, связанные с определением почти периодических на бесконечности функций.

Пусть $X$ - комплексное банахово пространство и $\mathbb{J}$-одно из множеств $\mathbb{R}$ или $\mathbb{R}_{+}$. Пусть $C_{b}(\mathbb{J}, X)$ - банахово пространство непрерывных ограниченных функций, определенных на $\mathbb{J}$ со значениями в комплексном банаховом пространстве $X$. Пусть $C_{b, u}(\mathbb{J}, X)$ - замкнутое подпространство равномерно непрерывных ограниченных функций. Через $C_{0}(\mathbb{J}, X)$ обозначим (замкнутое) подпространство функций $x \in C_{b}$, исчезающих на бесконечности, т.е.

$$
\lim _{|t| \rightarrow \infty}\|x(t)\|=0, \quad x \in C_{b}(\mathbb{J}, X) .
$$

В пространстве $C_{b}(\mathbb{J}, X)$ рассмотрим операторы сдвига

$$
S(t): C_{b}(\mathbb{J}, X) \rightarrow C_{b}(\mathbb{J}, X), \quad(S(t) x)(\tau)=x(\tau+t), \quad \tau \in \mathbb{J}, \quad t \in \mathbb{J}, \quad x \in C_{b}(\mathbb{J}, X) .
$$

Определение 2.1. Функцию $x$ из $C_{b, u}(\mathbb{J}, X)$ назовем интегрально убывающей на бесконечности, если

$$
\lim _{\alpha \rightarrow \infty} \frac{1}{\alpha} \sup _{t \in \mathbb{J}} \int_{0}^{\alpha}\|x(t+s)\| d s=0 .
$$

Обозначим символом $C_{0, \text { int }}=C_{0, \text { int }}(\mathbb{J}, X)$ множество интегрально убывающих на бесконечности функций. В частности, к таким подпространствам относится семейство замкнутых в $C_{b, u}(\mathbb{J}, X)$ подпространств

$$
C_{0, p}=C_{0, p}(\mathbb{J}, X)=\left\{x \in C_{b, u}(\mathbb{J}, X): \lim _{t \rightarrow \infty} \frac{1}{\alpha} \sup _{t \in \mathbb{J}} \int_{0}^{\alpha}\|x(s+t)\|^{p} d s=0\right\},
$$

где $p \in[1, \infty)$. Таким образом, $C_{0,1}=C_{0, \text { int }}$ - подпространство интегрально убывающих на бесконечности функций.

Определение 2.2. Обозначим символом $\mathcal{C}_{0}=\mathcal{C}_{0}(\mathbb{J}, X)$ замкнутое $\left(\right.$ с нормой из $\left.C_{b, u}\right)$ подпространство функций из $C_{b, u}(\mathbb{J}, X)$, обладающих следующими свойствами:

(1) $S(t) x \in \mathcal{C}_{0}$ для любого $t \in \mathbb{J}$ и любой функции $x \in \mathcal{C}_{0}$;

(2) $C_{0} \subset \mathcal{C}_{0} \subset C_{0 \text {,int }}$;

(3) $e_{\lambda} x \in \mathcal{C}_{0}$ для любого $\lambda \in \mathbb{R}$, где $e_{\lambda}(t)=e^{i \lambda t}, t \in \mathbb{R}$.

Введенный класс является более широким по сравнению с классом почти периодических на бесконечности функций, введенным в работах А. Г. Баскакова (см. [2,3]).

Определение 2.3. Функция $x \in C_{b, u}(\mathbb{J}, X)$ называется функиией, медленно меняющейся на бесконечности относительно подпространства $\mathcal{C}_{0}$, если для каждого $\alpha \in \mathbb{J}$ выполнено $S(\alpha) x-$ $x \in \mathcal{C}_{0}$.

Следует заметить, что в теории дифференциальных уравнений (см. [7]) приводится определение стационарной на бесконечности функции, которое эквивалентно определению медленно меняющейся функции.

Отметим что в $[3,4]$ давалось определение медленно меняющейся функции с использованием подпространства $C_{0}=C_{0}(\mathbb{R}, X)$. Свойства медленно меняющихся функций относительно подпространства $C_{0}$ также были отмечены в $[3,4,10,11,14]$. 
Множество всех медленно меняющихся на бесконечности функций из $C_{b, u}(\mathbb{J}, X)$ относительно подпространства $C_{0, \text { int }}$ будем обозначать через $C_{\mathrm{sl} \text {,int }}(\mathbb{J}, X)$ (см. $\left.[13]\right)$ и через $C_{\mathrm{sl}}(\mathbb{J}, X)$ - относительно подпространства $C_{0}$ (см. [3]). Символом $\mathcal{C}_{\mathrm{sl}, \mathcal{C}_{0}}$ будем обозначать подпространство, обладающее свойством

$$
C_{\mathrm{sl}}(\mathbb{J}, X) \subset \mathcal{C}_{\mathrm{sl}, \mathcal{C}_{0}} \subset C_{\mathrm{sl}, \text { int }}(\mathbb{J}, X) .
$$

Непосредственно из определения следует, что $C_{\mathrm{sl}}(\mathbb{J}, X)$ является замкнутым подпространством из $C_{b, u}(\mathbb{J}, X)$, инвариантным относительно сдвигов функций.

Приведем примеры медленно меняющихся на бесконечности функций из $C_{\mathrm{sl}, \mathcal{C}_{0}}$ для $X=\mathbb{C}$ :

(1) $x_{1}(t)=\sin \ln (1+|t|), t \in \mathbb{J} ;$

(2) $x_{2}(t)=c+x_{0}(t), t \in \mathbb{J}$, где $c \in \mathbb{C}$ и $x_{0}-$ любая функция из $\mathcal{C}_{0}(\mathbb{J})$;

(3) $x_{3}(t)=\operatorname{arctg} t, t \in \mathbb{J}$;

(4) любая непрерывно дифференцируемая функция $x \in C_{b}(\mathbb{J})$, обладающая свойством

$$
\lim _{|t| \rightarrow \infty} \dot{x}(t)=0 .
$$

Определение 2.4. Пусть $\varepsilon>0$. Число $\omega \in \mathbb{J}$ называется $\varepsilon$-периодом функции $x \in C_{b, u}(\mathbb{J}, X)$ на бесконечности относительно подпространства $\mathcal{C}_{0}(\mathbb{J}, X)$ исчезающих на бесконечности функций, если существует такая функция $x_{0} \in \mathcal{C}_{0}$, что

$$
\left\|S(\omega) x-x-x_{0}\right\|<\varepsilon .
$$

Множество $\varepsilon$-периодов функции $x \in C_{b, u}(\mathbb{J}, X)$ обозначим через $\Omega_{\infty}\left(x ; \mathcal{C}_{0} ; \varepsilon\right)$.

Если $\mathcal{C}_{0}=C_{0}(\mathbb{J}, X)$, то определение $\varepsilon$-периода функции из $C_{b, u}(\mathbb{J}, X)$ относительно $\mathcal{C}_{0}$ эквивалентно следующему определению.

Определение 2.5. Пусть $\varepsilon>0$. Число $\omega \in \mathbb{J}$ называется $\varepsilon$-периодом функиии $x \in C_{b, u}(\mathbb{J}, X)$ на бесконечности относительно подпространства $C_{0}(\mathbb{J}, X)$, если существует такое число $\alpha(\varepsilon) \geqslant 0$, что

$$
\sup _{|t| \geqslant \alpha(\varepsilon)}\|x(t+\omega)-x(t)\|<\varepsilon .
$$

Определение 2.6. Подмножество $\Omega$ из $\mathbb{J}$ называется относительно плотным на $\mathbb{J}$, если существует такое $l>0$, что $[t, t+l] \cap \Omega \neq \varnothing$ для любого $t \in \mathbb{J}$.

Определение 2.7 (аналог классического определения Бора). Функция $x$ из $C_{b, u}(\mathbb{J}, X)$ называется почти периодической на бесконечности относительно подпространства $\mathcal{C}_{0}$ исчезающих на бесконечности функций, если для любого $\varepsilon>0$ множество $\Omega_{\infty}\left(x ; \mathcal{C}_{0} ; \varepsilon\right)$ относительно плотно на $\mathbb{J}$.

Из определений $2.5,2.7$ следует, что каждая непрерывная функция $x \in C_{b, u}(\mathbb{R}, X)$, почти периодическая по Бору (в обычном смысле; см. [8]), является почти периодической на бесконечности относительно любого подпространства $\mathcal{C}_{0}$ исчезающих на бесконечности функций.

Множество классических почти периодических функций обозначим символом $A P(\mathbb{R}, X)$, а множество почти периодических на бесконечности функций относительно подпространства $\mathcal{C}_{0}=$ $\mathcal{C}_{0}(\mathbb{J}, X)$ - символом $A P_{\infty}\left(\mathbb{J}, X ; \mathcal{C}_{0}\right)$.

Непосредственно из определения 2.3 медленно меняющейся на бесконечности функции следует, что если $\Omega_{\infty}\left(x ; \mathcal{C}_{0} ; \varepsilon\right)=\mathbb{J}$ для любого $\varepsilon>0$, то $x \in \mathcal{C}_{\mathrm{sl}, \mathcal{C}_{0}}(\mathbb{J}, X)$. Таким образом, имеет место включение

$$
\mathcal{C}_{\mathrm{sl}, \mathcal{C}_{0}}(\mathbb{J}, X) \subset A P_{\infty}\left(\mathbb{J}, X, \mathcal{C}_{0}\right) .
$$

Определение 2.8. Множество функций $\mathcal{M} \subset C_{b, u}(\mathbb{J}, X)$ называется предкомпактным на бесконечности относительно подпространства $\mathcal{C}_{0}=\mathcal{C}_{0}(\mathbb{J}, X)$ исчезающих на бесконечности функций, если для любого $\varepsilon>0$ существует конечное число таких функций $b_{1}, \ldots, b_{N}$ из $\mathcal{M}(\varepsilon$-сеть на бесконечности), что для любой функции $x \in \mathcal{M}$ существуют функция $b_{k}, k \in\{1, \ldots, N\}$, и функция $\alpha_{\varepsilon} \in \mathcal{C}_{0}$, для которых имеет место оценка

$$
\left\|x-b_{k}-\alpha_{\varepsilon}\right\|<\varepsilon .
$$


Определение 2.9 (аналог определения Бохнера). Функция $x \in C_{b, u}(\mathbb{J}, X)$ называется почти периодической на бесконечности относительно подпространства $\mathcal{C}_{0}=\mathcal{C}_{0}(\mathbb{J}, X)$ исчезающих на бесконечности функций, если множество ее сдвигов $S(t) x, t \in \mathbb{J}$, является предкомпактным на бесконечности множеством относительно подпространства $\mathcal{C}_{0}$.

Определение 2.10 (аппроксимационное). Функция $x \in C_{b, u}(\mathbb{J}, X)$ называется почти периодической на бесконечности относительно подпространства $\mathcal{C}_{0}$ исчезающих на бесконечности функций, если для любого $\varepsilon>0$ можно указать конечное число таких вещественных чисел $\lambda_{1}, \ldots, \lambda_{N}$ и функций $x_{1}, \ldots, x_{N}$ из пространства $C_{\mathrm{sl}, \mathcal{C}_{0}}$ медленно меняющихся на бесконечности функций, что

$$
\sup _{t \in \mathbb{J}}\left\|x(t)-\sum_{k=1}^{N} x_{k}(t) e^{i \lambda_{k} t}\right\|<\varepsilon
$$

В работе будет использоваться понятие банахова модуля (банахова $L^{1}(\mathbb{R})$-модуля; см. $\left.[2,3]\right)$ над алгеброй суммируемых на $\mathbb{R}$ классов комплексных функций с нормой

$$
\|f\|_{1}=\int_{-\infty}^{+\infty}|f(t)| d t, \quad f \in L^{1}(\mathbb{R})
$$

где в роли мультипликативной операции выступает классическая свертка функций

$$
(f * g)(t)=\int_{-\infty}^{+\infty} f(s) g(t-s) d s, \quad t \in \mathbb{R}, \quad f, g \in L^{1}(\mathbb{R}) .
$$

Пусть $T: \mathbb{R} \rightarrow$ End $\mathcal{X}$ - сильно непрерывное изометрическое представление, где $\mathcal{X}$ - комплексное банахово пространство. Далее $\mathcal{X}$ рассматривается как $L^{1}(\mathbb{R})$-модуль. Будем говорить что модульная структура $\mathcal{X}$ ассочиирована с представлением $T$ (см. [6]), если имеют место равенства

$$
T(t)(f x)=(S(t) f) x=f T(t) x, \quad t \in \mathbb{R}
$$

и

$$
f(g x)=(f * g) x=(g * f) x
$$

для любых $f, g$ из алгебры $L^{1}(\mathbb{R})$ и любого вектора $x \in \mathcal{X}$. При этом

$$
\|f x\|=\left\|\int_{-\infty}^{+\infty} f(s) T(-s) x d s\right\| \leqslant \int_{-\infty}^{+\infty}\left|f(s)\|\| T(-s) x\left\|d s \leqslant \int_{-\infty}^{+\infty}|f(s)| d s\right\| x\|=\| f\left\|_{1}\right\| x \|\right.
$$

для любых $f \in L^{1}(\mathbb{R})$ и $x \in \mathcal{X}$. Далее для банахова $L^{1}(\mathbb{R})$-модуля $\mathcal{X}$ будет использоваться обозначение $(\mathcal{X}, T)$.

Если $T: \mathbb{R} \rightarrow$ End $\mathcal{X}$ - сильно непрерывная группа изометрий из алгебры End $\mathcal{X}$, то формула

$$
f x=\int_{-\infty}^{+\infty} f(s) T(-s) x d s, \quad f \in L^{1}(\mathbb{R}), \quad x \in \mathcal{X}
$$

определяет структуру банахова $L^{1}(\mathbb{R})$-модуля, ассоциированного с данным представлением $T$.

Обозначим через $\mathcal{X}_{c}$ множество всех $T$-непрерывных векторов из банахова $L^{1}(\mathbb{R})$-модуля $\mathcal{X}$.

Для каждой функции $f$ из алгебры $L^{1}(\mathbb{R})$ ее преобразование Фурье $\widehat{f}: \mathbb{R} \rightarrow \mathbb{C}$ определяется формулой

$$
\widehat{f}(\lambda)=\int_{\mathbb{R}} f(t) e^{-i \lambda t} d t, \quad \lambda \in \mathbb{R} .
$$

Определение 2.11. Спектром Берлинга вектора $x \in \mathcal{X}$ называется множество вещественных чисел $\Lambda(x)$ вида

$$
\Lambda(x)=\left\{\lambda_{0} \in \mathbb{R}: \quad f x \neq 0 \text { для любой функции } f \in L^{1}(\mathbb{R}) \text {, для которой } \widehat{f}\left(\lambda_{0}\right) \neq 0\right\} .
$$


Из определения следует, что

$$
\Lambda(x)=\mathbb{R} \backslash\left\{\mu_{0} \in \mathbb{R}: \text { существует такая функция } f \in L^{1}(\mathbb{R}) \text {, что } \widehat{f}\left(\mu_{0}\right) \neq 0 \text { и } f x=0\right\} .
$$

Имеют место следующие свойства спектра Берлинга векторов из банахова $L^{1}(\mathbb{R})$-модуля.

Лемма 2.1. Пусть $x \in X u f \in L^{1}(\mathbb{R})$. Тогда справедливы следующие утверждения.

(1) множество $\Lambda(x)$ замкнуто и $\Lambda(x)=\emptyset$ тогда и только тогда, когда $x=0$;

(2) $\Lambda(f x) \subseteq(\operatorname{supp} \widehat{f}) \cup \Lambda(x)$;

(3) $f x=0$, если $(\operatorname{supp} \widehat{f}) \cap \Lambda(x)=\emptyset, u f x=x$, если множество $\Lambda(x)$ компактно и $\widehat{f}=1$ в некоторой его окрестности;

(4) множество $\Lambda(x)$ одноточечно $\left(\Lambda(x)=\left\{\lambda_{0}\right\}\right)$, если и только если $x \neq 0$ u $T(t) x=e^{i \lambda_{0} t} x$, $t \in \mathbb{R}$.

Далее символом $\mathcal{X}$ будем обозначим фактор-пространство $C_{b, u}(\mathbb{J}, X) / \mathcal{C}_{0}(\mathbb{J}, X)$, являющееся банаховым пространством с нормой

$$
\|\widetilde{x}\|=\inf _{y \in x+\mathcal{C}_{0}}\|y\|
$$

где $\widetilde{x}=x+\mathcal{C}_{0}, x \in C_{b, u}(\mathbb{J}, X)$ - класс эквивалентности. В пространстве $\mathcal{X}$ определим сильно непрерывную группу изометрий $\widetilde{S}: \mathbb{R} \rightarrow$ End $\mathcal{X}$ формулой

$$
\widetilde{S}(t) \widetilde{x}=\widetilde{S(t) x}, \quad t \in \mathbb{R}, \quad \widetilde{x} \in \mathcal{X},
$$

где $S(t) x$-левый сдвиг функции $x$ для $t \geqslant 0$. Для $t<0$ символ $\widetilde{S(t) x}$ обозначает класс эквивалентности, содержащий функцию $x_{t} \in C_{b, u}(\mathbb{R}, X)$ вида

$$
x_{t}(s)= \begin{cases}x(s+t), & s+t>0, \\ -t^{-1} x(0)(s), & s+t \leqslant 0, s \geqslant 0 .\end{cases}
$$

Лемма 2.2. Представление $\widetilde{S}: \mathbb{R} \rightarrow$ End $\mathcal{X}$ сильно непрерывно и изометрично:

$$
\|\widetilde{S}(t) \widetilde{x}\|=\|\widetilde{x}\|, \quad x \in C_{b, u}(\mathbb{J}, X), \quad t \in \mathbb{R} .
$$

Доказательство. Докажем равенство (1) при $\mathbb{J}=\mathbb{R}_{+}$(при $\mathbb{I}=\mathbb{R}$ утверждение очевидно). Так как

$$
x_{t}(s)-x(s)= \begin{cases}x(s+t)-x(s) & \text { при } t \geqslant 0, \\ -\frac{s x(0)}{t}+\frac{(s-t) x(0)}{t} & \text { при } t \leqslant 0,\end{cases}
$$

то функция $t \mapsto \widetilde{S(t) x}: \mathbb{R} \rightarrow X$ непрерывна. Представление $\widetilde{S}: \mathbb{R} \rightarrow$ End $\mathcal{X}$ изометрично, так как

$$
\|\widetilde{S}(t) \widetilde{x}\|=\inf _{v_{0} \in \mathcal{C}_{0}} \sup _{s \in \mathbb{R}}\left\|y(s+t)-v_{0}(s)\right\|=\inf _{v_{0} \in \mathcal{C}_{0}} \sup _{s \in \mathbb{R}}\left\|y(s)-v_{0}(s)\right\|=\|\widetilde{x}\| .
$$

Теперь дадим последнее (четвертое) определение почти периодической на бесконечности функции.

Определение 2.12. Функция $x \in C_{b, u}(\mathbb{J}, X)$ называется почти периодической на бесконечноcmu относительно подпространства $\mathcal{C}_{0}$ исчезающих на бесконечности функций, если класс эквивалентности $\widetilde{x}=x+\mathcal{C}_{0}$ является почти периодическим вектором в пространстве $\mathcal{X}$ относительно изометрического представления $\widetilde{S}: \mathbb{R} \rightarrow \operatorname{End} \mathcal{X}$.

Теорема 2.1. Все определения почти периодической на бесконечности функиии (определения 2.7, 2.9, 2.10, 2.12) эквивалентны.

Доказательство. Рассмотрим фактор-пространство $\mathcal{X}=C_{b, u}(\mathbb{J}, X) / \mathcal{C}_{0}(\mathbb{J}, X)$ и определенную выше группу изометрий $T=\widetilde{S}: \mathbb{R} \rightarrow$ End $\mathcal{X}$. Для этого представления определение 2.9 соответствует свойству (4) из определения почти периодического вектора (см. [1, определение 14]). Поскольку все свойства из указанного определения эквивалентны, достаточно показать, что первые три его свойства эквивалентны определениям $2.7,2.9$ и 2.10 соответственно. 
Пусть $x \in C_{b, u}(\mathbb{J}, X)$, а $\widetilde{x} \in \mathcal{X}$ - класс эквивалентности, построенный по функции $x$. Тогда для любого $\varepsilon>0$ множество $\Omega_{\infty}\left(x ; \mathcal{C}_{0} ; \varepsilon\right) \cup\left(-\Omega_{\infty}\left(x ; \mathcal{C}_{0} ; \varepsilon\right)\right)$ совпадает с множеством $\Omega(\widetilde{x}, \varepsilon) \varepsilon$-периодов класса $\widetilde{x}$. Следовательно, соответствующие определения эквивалентны.

Эквивалентность определения 2.9 и свойства (2) определения 14 из [1] непосредственно следует из определения фактор-модуля $\mathcal{X}=C_{b, u}(\mathbb{J}, X) / \mathcal{C}_{0}(\mathbb{J}, X)$.

Докажем эквивалентность аппроксимационного определения 2.10 и свойства (3) из [1, определение 14]. Для доказательства достаточно установить, что спектр Берлинга $\Lambda(\widetilde{y})$ класса эквивалентности $\widetilde{y} \in \mathcal{X}, \widetilde{y}=y+\mathcal{C}_{0}$, является одноточечным множеством $\left(\Lambda(\widetilde{y})=\left\{\lambda_{0}\right\}\right)$ тогда и только тогда, когда функцию $y \in C_{b, u}(\mathbb{J}, X)$ можно представить в виде $y(t)=y_{0}(t) e^{i \lambda_{0} t}, t \in \mathbb{J}$, где $y_{0} \in \mathcal{C}_{\mathrm{sl}, \mathcal{C}_{0}}$.

Если $\Lambda(\widetilde{y})=\left\{\lambda_{0}\right\}$, то $\widetilde{S}(t) \widetilde{y}=e^{i \lambda_{0} t} \widetilde{y}$ для любого $t \in \mathbb{J}$ (см. [1, лемма 3, свойство 4]). Следовательно, $\Lambda\left(\widetilde{y}_{0}\right)=\{0\}$, где $y_{0}(s)=y(s) e^{-i \lambda_{0} s}, s \in \mathbb{J}$, и поэтому $\widetilde{S}(t) \widetilde{y}_{0}=\widetilde{y}_{0}$ для любого $t \in \mathbb{J}$. Таким образом, $S(t) y_{0}-y_{0} \in \mathcal{C}_{0}(\mathbb{J}, X), t \in \mathbb{J}$, т.е. $y_{0} \in \mathcal{C}_{\mathrm{sl}, \mathcal{C}_{0}}$.

Обратно, если $y(t)=y_{0}(t) e^{i \lambda_{0} t}, t \in \mathbb{J}$, где $y_{0} \in \mathcal{C}_{0}(\mathbb{J}, X)$, то $\widetilde{S}(t) \widetilde{y}=e^{i \lambda_{0} t} \widetilde{y}, t \in \mathbb{J}$, и поэтому в силу $\left[1\right.$, лемма 3 , свойство 4]) получим, что $\Lambda(\widetilde{y})=\left\{\lambda_{0}\right\}$. Теорема доказана.

3. Почти периодические на бесконечности решения разностных уравнений. Рассмотрим разностное уравнение вида

$$
x(t+1)=A x(t)+f(t), \quad t \in \mathbb{R},
$$

где $f \in \mathcal{C}_{0}(\mathbb{R}, X)$ и $A \in$ End $X$.

Лемма 3.1. Пусть спектральный радиус оператора $A$ из уравнения (2) удовлетворяет условию $r(A)=\max _{\lambda \in \sigma(A)}|\lambda|<1$. Тогда каждое ограниченное решение $x: \mathbb{R} \rightarrow X$ уравнения (2) принадлежит пространству $\mathcal{C}_{0}(\mathbb{R}, X)$, единственно и имеет вид

$$
x_{0}=\sum_{n=0}^{\infty} A^{n} S(-n-1) f, \quad f \in \mathcal{C}_{0}(\mathbb{R}, X) .
$$

Доказательство. Разностное уравнение (2) представим в виде

$$
x=A S(-1) x+S(-1) f, \quad(I-\widetilde{A}) x=S(-1) f,
$$

где $\widetilde{A}=A S(-1) \in$ End $C_{b, u}$. Так как в пространстве $C_{b, u}$ обратимая изометрия $S(-1)$ перестановочна с оператором умножения на оператор $A$, то

$$
r(\widetilde{A})=r(A)<1 .
$$

Следовательно (см. [16]), $I-A \in \operatorname{End} C_{b, u}$ непрерывно обратим, и обратный имеет вид

$$
(I-\widetilde{A})^{-1} y=\sum_{n=0}^{\infty} \widetilde{A}^{n} y=\sum_{n=0}^{\infty} A^{n} S(-n) y, y \in C_{b, u} .
$$

В частности, уравнение (2) имеет единственное решение $x_{0}: \mathbb{R} \rightarrow X$, которое представимо в виде (3). Очевидно, что $x_{0} \in \mathcal{C}_{0}(\mathbb{R}, X)$.

Лемма 3.2. Пусть оператор $A$ из уравнения (2) обратим и $r\left(A^{-1}\right)<1$. Тогда каждое ограниченное решение $x: \mathbb{R} \rightarrow X$ уравнения (2) принадлежит пространству $\mathcal{C}_{0}(\mathbb{R}, X)$, единственно и имеет вид

$$
x_{0}=-\sum_{n=0}^{\infty} A^{-n-1} S(n) f, \quad f \in \mathcal{C}_{0}(\mathbb{R}, X) .
$$

Доказательство. Применим оператор $A^{-1}$ к уравнению (2)

$$
x=A^{-1} S(1) x-A^{-1} f, \quad(I-\widetilde{A} x)=-A^{-1} f,
$$


где $\widetilde{A}=A^{-1} S(1)$. Следуя доказательству леммы 3.1, получим, что оператор $(I-\widetilde{A})$ обратим, и обратный имеет вид

$$
(I-\widetilde{A})^{-1}=-\sum_{n=0}^{\infty} A^{-n-1} S(n) .
$$

Поэтому ограниченное решение уравнения (2) представимо в виде

$$
x_{0}=-\sum_{n=0}^{\infty} A^{-n-1} S(n) f,
$$

где $f \in \mathcal{C}_{0}(\mathbb{R}, X)$.

Теорема 3.1. Пусть для $A \in$ End $X$ выполнено условие $\sigma(A) \cap \mathbb{T}=\left\{\gamma_{0}, \gamma_{2}, \ldots, \gamma_{N}\right\}$, где $\gamma_{k}=$ $e^{i \lambda_{k}}, 0 \leqslant k \leqslant N, u \mathbb{T}=\{\lambda \in \mathbb{C}:|\lambda|=1\}$. Если существует равномерно непрерывное ограниченное решение $x: \mathbb{R} \rightarrow X$ уравнения (2), то оно является почти периодической на бесконечности функцией $x \in A P_{\infty}\left(\mathbb{R}, X, \mathcal{C}_{0}\right)$ вида

$$
x(t)=\sum_{k=0}^{n} x_{k}(t) e^{i \lambda_{k} t}, \quad t \in \mathbb{R},
$$

где $x_{k} \in C_{\mathrm{sl}, \mathcal{C}_{0}}(\mathbb{R}, X), 0 \leqslant k \leqslant N$.

Доказательство. Спектр оператора $\in$ End $X$ представим в виде

$$
\sigma(A)=\sigma_{0} \cup \sigma_{\text {in }} \cup \sigma_{\text {out }},
$$

где $\sigma_{\text {in }}=\{\lambda \in \sigma(A):|\lambda|<1\}$ - совокупность точек спектра оператора $A$, лежащих внутри окружности, $\sigma_{\text {out }}=\{\lambda \in \sigma(A):|\lambda|>1\}$ - совокупность точек спектра оператора $A$, лежащих вне окружности. В соответствии с этим разбиением спектра рассмотрим проекторы $\mathcal{P}_{0}, \mathcal{P}_{\text {in }}, \mathcal{P}_{\text {out }}$, которые соответственно построены по спектральным множествам $\sigma_{0}, \sigma_{\text {in }}, \sigma_{\text {out }}$. Таким образом,

$$
I=\mathcal{P}_{0}+\mathcal{P}_{\text {in }}+\mathcal{P}_{\text {out }}
$$

Эти проекторы индуцируют разложение

$$
X=X_{0} \oplus X_{\text {in }} \oplus X_{\text {out }}
$$

пространства $X$, где $X_{0}=\operatorname{Im} \mathcal{P}_{0}, X_{\text {in }}=\operatorname{Im} \mathcal{P}_{\text {in }}, X_{\text {out }}=\operatorname{Im} \mathcal{P}_{\text {out }}$. Эти подпространства являются инвариантными для оператора $B$. Введем обозначения $A_{0}=A\left|X_{0}, A_{\text {in }}=A\right| X_{\text {in }}, A_{\text {out }}=A \mid X_{\text {out }}$. Таким образом, $A=A_{0} \oplus A_{\text {in }} \oplus A_{\text {out }}$ относительно построенного разложения пространства $X$.

Применяя проектор $\mathcal{P}_{\text {in }}$ к обеим частям уравнения $(2)$, получим функцию $x_{\text {in }}=\mathcal{P}_{\text {in }} x$, удовлетворяющую равенству

$$
S(1) x_{\text {in }}(t)=A_{\text {in }} x_{\text {in }}(t)+f_{\text {in }}(t), \quad f_{\text {in }}=\mathcal{P}_{\text {in }}, \quad f \in \mathcal{C}_{0}, \quad t \in \mathbb{R} .
$$

Из (6) следует, что

$$
\left(I-A_{\text {in }} S(-1)\right) x_{\text {in }}=S(-1) f_{\text {in }} .
$$

Поскольку $\|S(-1)\|=1, A_{\text {in }} S(-1) x_{\text {in }}(t)=S(-1) A_{\text {in }} x_{\text {in }}(t), t \in \mathbb{R}$, и спектральный радиус $r\left(A_{\text {in }}\right)$ оператора $A_{\text {in }}$ меньше единицы, то оператор $I-A_{\text {in }} S(-1)$ обратим, и из (7) следует, что

$$
x_{\mathrm{in}}=\left(I-A_{\mathrm{in}} S(-1)\right)^{-1} S(-1) f_{\mathrm{in}}=\sum_{k=0}^{\infty} A_{\mathrm{in}}^{k} S(-k-1) f_{\mathrm{in}} .
$$

Ясно, что $x_{\mathrm{in}} \in \mathcal{C}_{0}(\mathbb{R}, X)$. Аналогичный результат получим при применении проектора $\mathcal{P}_{\text {out }} \mathrm{K}$ уравнению (2):

$$
\left(S(1) x_{\text {out }}\right)(t)=A_{\text {out }} x_{\text {out }}(t)+y_{\text {out }}(t), \quad y_{\text {out }}=\mathcal{P}_{\text {out }} f \in \mathcal{C}_{0} .
$$

Оператор $A_{\text {out }}$ обратим и

$$
\sigma\left(A_{\text {out }}^{-1}\right)=\left\{\frac{1}{\lambda}, \lambda \in \sigma_{\text {out }}\right\},
$$


т.е. его спектральный радиус меньше единицы. Используя перестановочность операторов $S_{N}$ и $A_{\text {out }}($ см. (8)), получим равенства

$$
S(1) A_{\text {out }}^{-1} x_{\text {out }}(t)=x_{\text {out }}(t)+A_{\text {out }}^{-1} f_{\text {out }}(t), \quad t \in \mathbb{R},
$$

или

$$
\left(I-S(1) A_{\text {out }}^{-1}\right) x_{\text {out }}(t)=-A_{\text {out }}^{-1} f_{\text {out }}(t), \quad t \in \mathbb{R}
$$

Таким образом,

$$
x_{\text {out }}=-\left(I-S(1) A_{\text {out }}^{-1}\right)^{-1} A_{\text {out }}^{-1} f_{\text {out }}=-\sum_{k=0}^{\infty}\left(A_{\text {out }}^{-1} S(1)\right)^{k} A_{\text {out }}^{-1} f_{\text {out }}, \quad f_{\text {out }} \in \mathcal{C}_{0} .
$$

Следовательно $x_{\text {out }} \in \mathcal{C}_{0}(\mathbb{R}, X)$.

Проектор $\mathcal{P}_{0}$ можно представить в виде

$$
\mathcal{P}_{0}=P_{0}+\cdots+P_{N}
$$

где $P_{k} \in$ End $X_{0}$ - проектор, и

$$
A P_{k}=\gamma_{k} P_{k},
$$

где $\left|\gamma_{k}\right|=1,0 \leqslant k \leqslant N$. Применяя проектор $\mathcal{P}_{0}$ к разностному уравнению $(2)$ и далее применяя проектор $P_{k}$, получим

$$
P_{k} x_{0}(t+1)=P_{k} A_{0} x_{0}(t)+P_{k} f_{0}(t), \quad 0 \leqslant k \leqslant N, \quad t \in \mathbb{R},
$$

где $x_{0}(t)=\mathcal{P}_{0} x(t)$ и $f_{0}(t)=\mathcal{P}_{0} f(t), t \in \mathbb{R}$. Тогда сделав замену $\widetilde{x_{k}}(t)=e^{-i \lambda_{k} t} x_{k}(t), t \in \mathbb{R}$, получим

$$
S(1) \widetilde{x}_{k}=\widetilde{x}_{k}+\widetilde{f}_{k} .
$$

Так как $f_{k}(t)=\mathcal{P}_{0} f_{0}(t), t \in \mathbb{R}$, то $f_{k} \in \mathcal{C}_{0}$ и поэтому $\widetilde{f}_{k}=0$. Так как

$$
S(1) \widetilde{x}_{k}-\widetilde{x}_{k} \in \mathcal{C}_{0},
$$

то $\widetilde{x}_{k}$ - медленно меняющаяся на бесконечности функция, а $x_{k}$ отличается от $\widetilde{x}_{k}$ на множитель $e^{i \lambda_{k} t}, t \in \mathbb{R}$. Следовательно, $x_{0} \in A P_{\infty}\left(\mathbb{R}, X ; \mathcal{C}_{0}\right)$. В итоге получаем, что функция $x$ представима в виде $x=x_{0}+x_{\mathrm{in}}+x_{\text {out }}$. Следовательно, $x \in A P_{\infty}\left(\mathbb{R}, X ; \mathcal{C}_{0}\right)$, и решение имеет вид

$$
x(t)=\sum_{k=1}^{n} x_{k}(t) e^{i \lambda_{k} t}, \quad t \in \mathbb{R} .
$$

\section{СПИСОК ЛИТЕРАТУРЫ}

1. Баскаков А. Г., Струкова И. И., Тришина И. А. Почти периодические на бесконечности решения дифференциальных уравнений с неограниченными операторными коэффициентами// Сиб. мат. ж. 2018. - 59, № 2 (348). - С. 293-308.

2. Баскаков А. Г. Теория представлений банаховых алгебр, абелевых групп и полугрупп в спектральном анализе линейных операторов // Совр. мат. Фундам. направл. - 2004. - 9. - С. 3-151.

3. Баскаков А. Г., Калужина Н. С. Теорема Берлинга для функций с существенным спектром из однородных пространств и стабилизация решений параболических уравнений// Мат. заметки. - 2012. 92, № 5. - С. 643-661.

4. Баскаков А. Г., Калужина Н. С., Поляков Д. М. Медленно меняющиеся на бесконечности полугруппы операторов// Изв. вузов. Мат. - 2014. - № 7. - С. 3-14.

5. Спектральные критерии почти периодичности решений функциональных уравнений// Мат. заметки. - 1978. - 24, № 2. - С. 195-206.

6. Гелъфанд И. М., Райков Д. А., Шилов Г. Е. Коммутативные нормированные кольца. - М.: Физматгиз, 1967.

7. Далецкий Ю. Л., Крейн М. Г. Устойчивость решений дифференциальных уравнений в банаховом пространстве. - М.: Наука, 1970.

8. Левитан Б. М. Почти периодические функции. - М.: Гостехиздат, 1953.

9. фон Нейман Дж. Избранные труды по функциональному анализу. - М.: Наука, 1987. 
10. Рыжкова А. А., Тришина И. А. О почти периодических на бесконечности решениях разностных уравнений// Изв. Саратов. ун-та. Сер. Мат. Мех. Информ. - 2015. - 15, № 1. - С. 45-49.

11. Рыжкова А. А., Тришина И. А. О периодических на бесконечности функциях// Науч. вед. Белгород. гос. ун-та. Сер. Мат. Физ. - 2014. - 36, № 19. - С. 71-75.

12. Соболев С. Л. О почти периодичности решений волнового уравнения// Докл. АН СССР. - 1945. 49, № 1. - C. 12-15.

13. Тришина И. А. Почти периодические на бесконечности функции относительно подпространства интегрально убывающих на бесконечности функций// Изв. Саратов. ун-та. Сер. Мат. Мех. Информ. 2017. - 17, № 4. - С. 402-418.

14. Тришина И. А. Медленно меняющиеся на бесконечности функции// Вестн. Воронеж. гос. ун-та. Сер. Физ. Мат. - 2017. - № 4. - С. 134-144.

15. Amerio L., Prouse G. Almost Periodic Functions and Functional Equations. - New York: Springer-Verlag, 1971.

16. Arendt W., Batty C. J. K. Asymptotic almost periodic solutions of inhomogeneous Cauchy problems on the half-line// Bull. London Math. Soc. - 1991. — 31, № 3. - P. 291-304.

17. Bochner S., von Neuman J. On compact solution of operational differentional equations// Ann. Math. 1935. - 36. - P. 435-447.

Высоцкая Ирина Алевтиновна

Воронежский государственный университет

E-mail: i.a.tishina@gmail.com 